\title{
Multi-Derivative Multistep Method for Initial Value Problems Using Boundary Value Technique
}

\author{
Emmanuel A. Areo, Oluwatoyin A. Edwin* \\ Department of Mathematical Sciences, The Federal University of Technology, Akure, Nigeria \\ Email: eaare@futa.edu.ng, *toyinedwin@yahoo.com
}

How to cite this paper: Areo, E.A. and Edwin, O.A. (2020) Multi-Derivative Multistep Method for Initial Value Problems Using Boundary Value Technique. Open Access Library Journal, 7: e6063. https://doi.org/10.4236/oalib.1106063

Received: January 10, 2020

Accepted: March 16, 2020

Published: March 19, 2020

Copyright $\odot 2020$ by author(s) and Open Access Library Inc.

This work is licensed under the Creative Commons Attribution International License (CC BY 4.0).

http://creativecommons.org/licenses/by/4.0/

\section{(c) (i) Open Access}

\begin{abstract}
The second derivative method which is A-stable is derived using Interpolation Collocation approach. The continuous method obtained are used to generate the main method and complementary methods to solve initial value problems of ordinary differential equation via boundary value technique. Numerical result obtained via the methods shows that the new method can compete with the existing ones in the literature.
\end{abstract}

\section{Subject Areas}

Ordinary Differential Equation

\section{Keywords}

Collocation, Interpolation, Continuous Method, Chebyshev Polynomial, Stiff Ordinary Differential Equation

\section{Introduction}

Differential Equations (DEs) are important tools of solving real-life problems and a wide variety of natural phenomena are modeled into differential equations. DEs which normally arise in biological models, circuit theory models, circuit theory models, fluid and chemical kinetics models may or may not have exact solutions, thus a need for a numerical solution.

Consider the initial value problem of the form

$$
y^{\prime}=f(x, y), \quad y(a)=y_{0}, \quad x \in[a, b]
$$

Several numerical methods for the solution of (1) were proposed such as Tau method (Onumanyi [1]) and the Finite Difference method (Fox [2]). Interpolation 
collocation methods have been proposed for the numerical solution of Equation (1). Multiderivative method for solving systems of ODE was proposed by Obrenchkoff [3] and special cases of the Obrenchkoff were later proposed by Enright [3], Cash [4], Jia-Xiang, Jiao-Xun in recent article of Ehigie, Jator, Sofoluwe and Okunuga [5]. And of these methods, the justification for including higher term in such method was clearly stated by Enright [3], which will include method with higher order, obtain stability at infinity and obtain a method with reasonable stability properties on the neighborhood of the origin. This class of Enright's schemes is a special class of the Obrenchkoff cited in Ehigie [5] methods which are found to be of order $p=k+2$ for a $k$ step method.

In this paper, a continuous form of the second derivative multistep method shall be derived through a multistep collocation technique so that the method derived shall recover the Enright's second derivative method and other possible method. The method will be in block and will be implemented on IVP. This paper will further discuss the implementation of the newly developed methods using Boundary Value Methods as it was also implemented in Axelsson and Verwer [6], Amodio and Mazzia [7], Brugnano and Trigiante [8], Jator and Sahi [9], Ehigie [5], so that we can obtain the numerical solution simultaneously. The advantages of this approach are that the global error at the end of the integration is smaller than the accumulation of the various local truncation error obtained via step by step implementation (Lambert [10]), more also the block method is known to relax the A-stability criteria of other higher methods (Axelsson and Verwer [6]).

\section{Theoretical Procedure}

The second derivative multistep methods are derived using collocation technique as discussed in Ehigie [5].

The general second derivative formula for solving Equation (1) using $k$-step second derivative linear multistep method is of the form.

$$
\sum_{j=0}^{k} \alpha_{j} y_{n+j}=h \sum_{j=0}^{k} \beta_{j} f_{n+j}+h^{2} \sum_{j=0}^{k} \delta_{j} g_{n+j}
$$

where $y_{n+j} \approx y\left(x_{n}+j h\right)$,

$$
\begin{gathered}
f_{n+j} \equiv f\left(x_{n}+j h, y\left(x_{n}+j h\right)\right) \\
g_{n+j}=\left.\frac{\mathrm{d} f(x, y(x))}{\mathrm{d} x}\right|_{y=y_{n+j}} ^{x=x_{n+j}}
\end{gathered}
$$

$x_{n}$ is a discrete point at $n, \alpha_{j}, \beta_{j}$ and $\gamma_{j}$ are coefficients to be determined. To obtained the method of the form (1), $y(x)$ is approximated by (2) where $T_{n}$ a chebyshev polynomial of the form

$$
y(x)=\sum_{n=0}^{r} a_{n} T_{n}(x) .
$$


Equation (3) will be considered for the derivation of the main and complementary methods for the two classes of continuous second derivatives multistep method of Enright which is a special case of (2).

Interpolating $y(x)$ at point $x_{n+k-1}$, collocating $y^{\prime}(x)$ at points $x_{n+j}$, $j=0,1,2, \cdots, k$ and collocating $y^{\prime \prime}(x)$ at point $x_{n+k}$, i.e.

$$
\begin{gathered}
x_{n+k-1}=y_{n+k-1} \\
y^{\prime}(x)=f_{n+j} \\
y^{\prime \prime}(x)=g_{n+j}, \quad j=0,1,2, \cdots, k
\end{gathered}
$$

The system of equations generated are solved to obtained the coefficients of $a_{j}, j=0,1,2, \cdots, k+4$ which are used to generate the continous multistep method of Enright of the form

$$
y(x)=y_{n+k-1}+h \sum_{j=0}^{k} \beta_{j} f_{n+j}+h^{2} \delta_{j} g_{n+k}
$$

Evaluating (4) at $x=x_{n+k}$ yields the second drivative multistep method of Enright also evaluating at $x=x_{n+j}, j=0,1,2, \cdots, k-2$ gives $(k-1)$ methods, which will be called complementary methods to complete the $\mathrm{k}$ block for the system. The Enright's method is obtained in the form.

$$
y_{n+k}=y_{n+k-1}+h \sum_{j=0}^{k} \beta_{j}(x) f_{n+j}+h^{2} \gamma_{j} g_{n+k}
$$

\section{Specification of the Derived Methods}

\subsection{Four-Step Method}

The objective is to derive the multi-derivative main method of the form:

$$
\sum_{j=3}^{4} \alpha_{j} y_{n+j}=h \sum_{j=0}^{4} \beta_{j} f_{n+j}+h^{2} \gamma_{j} g_{n+4}
$$

where $\alpha_{j}, \beta_{j}, \gamma_{j}$ are coefficients. In order to obtain Equation (6), we proceed by seeking an approximation to the exact solution by assuming a continuous solution $y(x)$ of the form

$$
y(x)=\sum_{j=0}^{6} a_{j} T_{j}(x)
$$

with first derivative given by

$$
f(x)=y^{\prime}(x)=\sum_{j=0}^{6} a_{j} T_{j}^{\prime}(x),
$$

with second derivative given by

$$
g(x)=y^{\prime \prime}(x)=\sum_{j=0}^{6} a_{j} T_{j}^{\prime \prime}(x),
$$

Interpolating (7) at $x=x_{n+3}$, collocating (8) at $x=x_{n+j}, j=0(1) 6$ and collocating (9) at $x=x_{n+4}$ where $n$ is the grid index using maple we obtain the coefficients $a_{j}^{\prime} s$ : 


$$
\begin{aligned}
a_{0}= & y_{n+3}-\frac{22729}{36864} h f_{n+3}-\frac{54275}{589824} h f_{n}-\frac{103817}{110592} h f_{n+1}-\frac{47411}{49152} h f_{n+2} \\
& +\frac{198005}{1769472} h f_{n+4}-\frac{6269}{147456} h^{2} g_{n+4}, \\
a_{1}= & \frac{7459}{49152} h f_{n}+\frac{1669}{3072} h f_{n+1}-\frac{1489}{4096} h f_{n+2}+\frac{899}{3072} h f_{n+3} \\
& -\frac{6103}{49152} h f_{n+4}+\frac{215}{4096} h^{2} g_{n+4}, \\
a_{2}= & \frac{3365}{73728} h f_{n+1}-\frac{731}{24576} h f_{n+3}+\frac{15811}{1179648} h f_{n+4}+\frac{955}{32768} h f_{n+2} \\
& -\frac{571}{98304} h^{2} g_{n+4}-\frac{22981}{393216} h f_{n}, \\
a_{3}= & \frac{961}{24576} h f_{n+2}+\frac{3181}{294912} h f_{n}-\frac{571}{18432} h f_{n+3}-\frac{589}{18432} h f_{n+1} \\
& +\frac{3847}{294912} h f_{n+4}-\frac{45}{8192} h^{2} g_{n+4}, \\
a_{4}= & \frac{91}{12288} h f_{n+3}+\frac{155}{36864} h f_{n+1}-\frac{1967}{589824} h f_{n+4}-\frac{119}{16384} h f_{n+2} \\
& +\frac{71}{49152} h^{2} g_{n+4}-\frac{199}{196608} h f_{n}, \\
a_{5}= & \frac{133}{491520} h f_{n+4}+\frac{19}{40960} h f_{n+2}-\frac{1}{8192} h^{2} g_{n+4}-\frac{17}{30720} h f_{n+3} \\
& -\frac{7}{30720} h f_{n+1}+\frac{23}{491520} h f_{n}, \\
a_{6}= & \frac{1}{294912} h^{2} g_{n+4}+\frac{1}{73728} h f_{n+3}+\frac{1}{221184} h f_{n+1}-\frac{1}{1179648} h f_{n} \\
& -\frac{25}{3538944} h f_{n+4} \\
&
\end{aligned}
$$

Substituting the values of $a_{j}^{\prime} s$ in the approximate solution (7), will generate a continuous form of the scheme of the form:

$$
\begin{aligned}
y(x)= & a_{0}+a_{1}(2 x-1)+a_{2}\left(8 x^{2}-8 x+1\right)+a_{3}\left(32 x^{3}-48 x^{2}+18 x-1\right) \\
& +a_{4}\left(128 x^{4}-256 x^{3}+160 x^{2}-32 x+1\right) \\
& +a_{5}\left(512 x^{5}-1280 x^{4}+1120 x^{3}-400 x^{2}+50 x-1\right) \\
& +a_{6}\left(2048 x^{6}-6144 x^{5}+6912 x^{4}-3584 x^{3}+840 x^{2}-72 x+1\right)
\end{aligned}
$$

On evaluating (10) at $x=x_{n+4}, x=x_{n+2}, x=x_{n+1}, x=x_{n}$

$$
\begin{aligned}
& y_{n+4}-y_{n+3} \\
= & h\left[-\frac{17}{5760} f_{n}+\frac{1}{45} f_{n+1}-\frac{41}{480} f_{n+2}+\frac{47}{90} f_{n+3}+\frac{3133}{5760} f_{n+4}\right]-h^{2} \frac{3}{32} g_{n+4} \\
y_{n+2}-y_{n+3} & \\
= & h\left[-\frac{11}{1920} f_{n}+\frac{7}{135} f_{n+1}-\frac{83}{160} f_{n+2}-\frac{19}{30} f_{n+3}+\frac{1831}{17280} f_{n+4}\right]-h^{2} \frac{11}{288} g_{n+4}
\end{aligned}
$$




$$
\begin{aligned}
& y_{n+1}-y_{n+3}=h\left[\frac{1}{90} f_{n}-\frac{17}{45} f_{n+1}-\frac{19}{15} f_{n+2}-\frac{17}{45} f_{n+3}+\frac{1}{90} f_{n+4}\right] \\
y_{n}-y_{n+3} & =h\left[-\frac{201}{640} f_{n}-\frac{7}{5} f_{n+1}-\frac{99}{160} f_{n+2}-\frac{9}{10} f_{n+3}+\frac{149}{640} f_{n+4}\right]-h^{2} \frac{3}{32} g_{n+4}
\end{aligned}
$$

Equations (11)-(14) will be taken as Boundary Value Method for $k=4$ denoted as BVM4. To implement (10), we use a modified block method defined as follows:

$$
h^{\lambda} \sum_{j=1}^{q} a_{i j} y_{n+j}^{\lambda}=h^{\lambda} \sum_{j=0}^{q} e_{i j} y_{n}^{\lambda}+h^{\mu-\lambda}\left[\sum_{j=1}^{q} d_{i j} f_{n}+\sum_{j=1}^{q} b_{i j} f_{n+j}\right]
$$

where $\lambda$ is the power of the derivative of the continuous method and $\mu$ is the order of the problem to be solved; $q=r+s$. In vector notation, (15) can be written as

$$
h^{\lambda} \bar{a} Y_{m}=h^{\lambda} \bar{e} y_{m}+h^{\mu-\lambda}\left[\bar{d} f\left(y_{m}\right)+\bar{b} F\left(Y_{m}\right)\right]
$$

The matrices $\bar{a}=\left(a_{i j}\right), \bar{b}=\left(b_{i j}\right), \bar{e}=\left(e_{i j}\right), \bar{d}=\left(d_{i j}\right)$ are constant coefficient matrices and $Y_{m}=\left(y_{n+v_{i}}, y_{n+1}, y_{n+v_{i}}^{\prime}, y_{n+1}^{\prime}\right)^{\mathrm{T}}$, $y_{m}=\left(y_{n-(r-1)}, y_{n-(r-2)}, \cdots, y_{n}\right)^{\mathrm{T}}, \bar{F}\left(Y_{m}\right)=\left(f_{n+v_{i}}, f_{n+j}\right)^{\mathrm{T}}$ and $f\left(y_{m}\right)=\left(f_{n-i}, \cdots, f_{n}\right), i=1, \cdots, q$. The normalized version of (16) is given by

$$
\bar{A} Y_{m}=h^{\lambda} \bar{E} y_{m}+h^{\mu-\lambda}\left[\bar{D} f\left(y_{m}\right)+\bar{B} F\left(Y_{m}\right)\right]
$$

The modified block formulae (15) and (16) are employed to simultaneously obtain value for $y_{n+1}, y_{n+2}, y_{n+3}, y_{n+4}$ needed to implement (11). i.e. Combining (11)-(14) in the form of (15) and (16), yield the block method below:

$$
\begin{aligned}
& \left(\begin{array}{rrrr}
1 & -1 & 0 & 0 \\
0 & -1 & 1 & 0 \\
0 & -1 & 0 & 1 \\
0 & -1 & 0 & 0
\end{array}\right)\left(\begin{array}{l}
y_{n+4} \\
y_{n+3} \\
y_{n+2} \\
y_{n+1}
\end{array}\right)=\left(\begin{array}{l}
0 \\
0 \\
0 \\
1
\end{array}\right)\left(y_{n}\right)+h\left(\begin{array}{cccc}
\frac{1}{45} & -\frac{41}{480} & \frac{47}{90} & \frac{3133}{5760} \\
\frac{7}{135} & -\frac{83}{160} & -\frac{19}{30} & \frac{1831}{17280} \\
-\frac{17}{45} & -\frac{19}{15} & -\frac{17}{45} & \frac{1}{90} \\
-\frac{7}{5} & -\frac{99}{160} & -\frac{9}{10} & \frac{149}{640}
\end{array}\right)\left(\begin{array}{l}
f_{n+1} \\
f_{n+2} \\
f_{n+3} \\
f_{n+4}
\end{array}\right) \\
& +h\left(\begin{array}{c}
-\frac{17}{5760} \\
-\frac{11}{1920} \\
\frac{1}{90} \\
-\frac{201}{640}
\end{array}\right)\left(f_{n}\right)+h^{2}\left(\begin{array}{c}
-\frac{3}{32} \\
-\frac{11}{288} \\
0 \\
-\frac{3}{32}
\end{array}\right)\left(g_{n+4}\right)
\end{aligned}
$$

using (16) to obtain the block solution as 


$$
\begin{aligned}
\left(\begin{array}{llll}
1 & 0 & 0 & 0 \\
0 & 1 & 0 & 0 \\
0 & 0 & 1 & 0 \\
0 & 0 & 0 & 1
\end{array}\right)\left(\begin{array}{l}
y_{n+4} \\
y_{n+3} \\
y_{n+2} \\
y_{n+1}
\end{array}\right)= & \left(\begin{array}{l}
1 \\
1 \\
1 \\
1
\end{array}\right)\left(y_{n}\right)+h\left(\begin{array}{cccc}
\frac{46}{45} & -\frac{311}{480} & \frac{47}{90} & -\frac{1277}{5760} \\
\frac{196}{135} & \frac{1}{10} & \frac{4}{15} & -\frac{137}{1080} \\
\frac{7}{5} & \frac{99}{160} & \frac{9}{10} & -\frac{149}{640} \\
\frac{64}{45} & \frac{8}{15} & \frac{64}{45} & \frac{14}{45}
\end{array}\right)\left(\begin{array}{l}
f_{n+1} \\
f_{n+2} \\
f_{n+3} \\
f_{n+4}
\end{array}\right) \\
& \left.+h\left(\begin{array}{l}
\frac{1873}{5760} \\
\frac{37}{120} \\
\frac{201}{640} \\
\frac{14}{45}
\end{array}\right)\left(\begin{array}{l}
\frac{3}{32} \\
\frac{1}{18} \\
\frac{3}{32} \\
0
\end{array}\right)+h_{n+4}^{2}\right)
\end{aligned}
$$

Written the above explicitly gives:

$$
\begin{gathered}
y_{n+1}=y_{n}+h\left[\frac{1873}{5760} f_{n}+\frac{46}{45} f_{n+1}-\frac{311}{480} f_{n+2}+\frac{47}{90} f_{n+3}-\frac{1277}{5760} f_{n+4}\right]+h^{2} \frac{3}{32} g_{n+4} \\
y_{n+2}=y_{n}+h\left[\frac{37}{120} f_{n}+\frac{196}{135} f_{n+1}+\frac{1}{10} f_{n+2}+\frac{4}{15} f_{n+3}-\frac{137}{1080} f_{n+4}\right]+h^{2} \frac{1}{18} g_{n+4} \\
y_{n+3}=y_{n}+h\left[\frac{201}{640} f_{n}+\frac{7}{5} f_{n+1}+\frac{99}{160} f_{n+2}+\frac{9}{10} f_{n+3}-\frac{149}{640} f_{n+4}\right]+h^{2} \frac{3}{32} g_{n+4} \\
y_{n+4}=y_{n}+h\left[\frac{14}{45} f_{n}+\frac{64}{45} f_{n+1}+\frac{8}{15} f_{n+2}+\frac{64}{45} f_{n+3}+\frac{14}{45} f_{n+4}\right]
\end{gathered}
$$

\subsection{Five-Step Method}

The objective is to derive the multi-derivative main method of the form:

$$
\sum_{j=4}^{5} \alpha_{j} y_{n+j}=h \sum_{j=0}^{5} \beta_{j} f_{n+j}+h^{2} \gamma_{j} g_{n+5}
$$

where $\alpha_{j}, \beta_{j}, \gamma_{j}$ are coefficients are to be determined. In order to obtain Equation (22), I proceed by seeking an approximation to the exact solution by assuming a continuous solution $y(x)$ of the form

$$
y(x)=\sum_{j=0}^{7} a_{j} T_{j}(x)
$$

with first derivative given by

$$
f(x)=y^{\prime}(x)=\sum_{j=0}^{7} a_{j} T_{j}^{\prime}(x),
$$

with second derivative given by

$$
g(x)=y^{\prime \prime}(x)=\sum_{j=0}^{7} a_{j} T_{j}^{\prime \prime}(x),
$$

Interpolating (23) at $x=x_{n+4}$, collocating (24) at $x=x_{n+j}, j=0(1) 5$ and 
collocating (25) at $x=x_{n+5}$ where $\mathrm{n}$ is the grid index using maple we obtain the coefficients $a_{j} \mathrm{~s}$ :

$$
\begin{aligned}
& a_{0}=y_{n+4}-\frac{463807}{368640} h f_{n+3}-\frac{316403}{3686400} h f_{n}-\frac{181051}{184320} h f_{n+1}-\frac{903161}{1105920} h f_{n+2} \\
& -\frac{265139}{737280} h f_{n+4}+\frac{13987}{5529600} h f_{n+5}+\frac{623}{184320} h^{2} g_{n+5} \text {, } \\
& a_{1}=\frac{237263}{1638400} h f_{n}+\frac{153505}{262144} h f_{n+1}-\frac{138739}{294912} h f_{n+2}+\frac{27969}{65536} h f_{n+3} \\
& -\frac{20081}{65536} h f_{n+4}+\frac{7059707}{58982400} h f_{n+5}-\frac{47011}{983040} h^{2} g_{n+5}, \\
& a_{2}=\frac{327}{8192} h f_{n+1}-\frac{47111}{819200} h f_{n}+\frac{717}{16384} h f_{n+2}-\frac{799}{16384} h f_{n+3} \\
& +\frac{1237}{32768} h f+n+4-\frac{6207}{409600} h f_{n+5}+\frac{251}{40960} h^{2} g_{n+5}, \\
& a_{3}=\frac{56431}{4915200} h f_{n}-\frac{9485}{262144} h f_{n+1}+\frac{44029}{884736} h f_{n+2}-\frac{8695}{196608} h f_{n+3} \\
& +\frac{2053}{65536} h f_{n+4}-\frac{2152541}{176947200} h f_{n+5}+\frac{14293}{2949120} h^{2} g_{n+5} \text {, } \\
& a_{4}=-\frac{509}{409600} h f_{n}+\frac{23}{4096} h f_{n+1}-\frac{89}{8192} h f_{n+2}+\frac{99}{8192} h f_{n+3} \\
& -\frac{153}{16384} h f_{n+4}+\frac{767}{204800} h f_{n+5}-\frac{31}{20480} h^{2} g_{n+5}, \\
& a_{5}=\frac{961}{983040} h f_{n+4}-\frac{1561}{3932160} h f_{n+1}-\frac{1603}{3932160} h f_{n+5}+\frac{443}{491520} h f_{n+2} \\
& +\frac{11}{65536} h^{2} g_{n+5}-\frac{1129}{983040} h f_{n+3}+\frac{73}{983040} h f_{n}, \\
& a_{6}=\frac{71}{3686400} h f_{n+5}-\frac{5}{147456} h f_{n+2}-\frac{1}{122880} h^{2} g_{n+5}+\frac{7}{147456} h f_{n+3} \\
& +\frac{1}{73728} h f_{n+1}-\frac{17}{7372800} h f_{n}-\frac{13}{294912} h f_{n+4}, \\
& a_{7}=\frac{1}{6881280} h^{2} g_{n+5}-\frac{1}{1376256} h f_{n+3}-\frac{1}{5505024} h f_{n+1}+\frac{1}{34406400} h f_{n} \\
& +\frac{1}{2064384} h f_{n+2}+\frac{1}{1376256} h f_{n+4}-\frac{137}{412876800} h f_{n+5}
\end{aligned}
$$

Substituting the values of $a_{j}^{\prime} s$ in the approximate solution (23), I obtain a continuous form of the scheme of the form:

$$
\begin{aligned}
y(x) & =a_{0}+a_{1}(2 x-1)+a_{2}\left(8 x^{2}-8 x+1\right)+a_{3}\left(32 x^{3}-48 x^{2}+18 x-1\right) \\
& +a_{4}\left(128 x^{4}-256 x^{3}+160 x^{2}-32 x+1\right) \\
& +a_{5}\left(512 x^{5}-1280 x^{4}+1120 x^{3}-400 x^{2}+50 x-1\right) \\
& +a_{6}\left(2048 x^{6}-6144 x^{5}+6912 x^{4}-3584 x^{3}+840 x^{2}-72 x+1\right) \\
& +a_{7}\left(8192 x^{7}-28672 x^{6}+39424 x^{5}-26880 x^{4}+9408 x^{3}-1568 x^{2}+98 x-1\right)
\end{aligned}
$$

On evaluating (26) at $x=x_{n+5}, x=x_{n+3}, x=x_{n+2}, x=x_{n+1}, x=x_{n}$ we 
generate the main methods and complementary methods which invariably complete the boundary value techniques.

$$
\begin{aligned}
y_{n+5}-y_{n+4}=h & {\left[\frac{41}{25200} f_{n}-\frac{529}{40320} f_{n+1}+\frac{373}{7560} f_{n+2}-\frac{1271}{10080} f_{n+3}\right.} \\
+ & \left.\frac{2837}{5040} f_{n+4}+\frac{317731}{604800} f_{n+5}\right]-h^{2} \frac{863}{10080} g_{n+5} \\
y_{n+3}-y_{n+4}= & h\left[\frac{19}{8400} f_{n}-\frac{89}{4480} f_{n+1}+\frac{677}{7560} f_{n+2}-\frac{1933}{3360} f_{n+3}\right. \\
& \left.-\frac{323}{560} f_{n+4}+\frac{48467}{604800} f_{n+5}\right]-h^{2} \frac{271}{10080} g_{n+5} \\
y_{n+2}-y_{n+4}= & h\left[-\frac{1}{630} f_{n}+\frac{53}{2520} f_{n+1}-\frac{382}{945} f_{n+2}-\frac{773}{630} f_{n+3}\right. \\
& \left.-\frac{263}{630} f_{n+4}+\frac{221}{7560} f_{n+5}\right]-\frac{1}{126} h^{2} g_{n+5} \\
= & {\left[\frac{33}{2800} h f_{n}-\frac{1737}{4480} f_{n+1}-\frac{337}{280} f_{n+2}-\frac{1023}{1120} f_{n+3}\right.} \\
& \left.-\frac{339}{560} f_{n+4}+\frac{2201}{22400} f_{n+5}\right]-\frac{39}{1120} h^{2} g_{n+5} \\
y_{n+1}-y_{n+4} & \left.-\frac{2}{35} f_{n+4}-\frac{548}{4725} f_{n+5}\right]+\frac{16}{315} h^{2} g_{n+5} \\
y_{n}-y_{n+4}= & {\left[-\frac{158}{525} f_{n}-\frac{52}{35} f_{n+1}-\frac{344}{945} f_{n+2}-\frac{176}{105} f_{n+3}\right.}
\end{aligned}
$$

Equations (27)-(31) will be taken as Boundary Value method for $k=5$ and denoted as BVM5. The modified block formulae (15) and (16) are employed to simultaneously obtain value for $y_{n+1}, y_{n+2}, y_{n+3}, y_{n+4}, y_{n+5}$ needed to implement (27), i.e., combining (27)-(31) in the form of (15) and (16), yield the block method below:

$$
\begin{aligned}
& \left(\begin{array}{lllll}
1 & -1 & 0 & 0 & 0 \\
0 & -1 & 1 & 0 & 0 \\
0 & -1 & 0 & 1 & 0 \\
0 & -1 & 0 & 0 & 1 \\
0 & -1 & 0 & 0 & 0
\end{array}\right)\left(\begin{array}{l}
y_{n+5} \\
y_{n+4} \\
y_{n+3} \\
y_{n+2} \\
y_{n+1}
\end{array}\right) \\
& =\left(\begin{array}{l}
0 \\
0 \\
0 \\
0 \\
1
\end{array}\right)\left(y_{n}\right)+h\left(\begin{array}{ccccc}
-\frac{529}{40320} & \frac{373}{7560} & -\frac{1271}{10080} & \frac{2837}{5040} & \frac{317731}{604800} \\
-\frac{89}{4480} & \frac{677}{7560} & -\frac{1933}{3360} & -\frac{323}{560} & \frac{48467}{604800} \\
-\frac{53}{2520} & -\frac{382}{945} & -\frac{773}{630} & -\frac{263}{630} & \frac{221}{7560} \\
-\frac{1737}{4480} & -\frac{337}{280} & -\frac{1023}{1120} & -\frac{339}{560} & \frac{2201}{22400} \\
-\frac{52}{35} & -\frac{344}{945} & -\frac{176}{105} & -\frac{2}{35} & -\frac{548}{4725}
\end{array}\right)\left(\begin{array}{l}
f_{n+5} \\
f_{n+4} \\
f_{n+3} \\
f_{n+2} \\
f_{n+1}
\end{array}\right)
\end{aligned}
$$




$$
+h\left(\begin{array}{c}
\frac{41}{25200} \\
\frac{19}{8400} \\
-\frac{1}{630} \\
\frac{33}{2800} \\
-\frac{158}{525}
\end{array}\right)\left(f_{n}\right)+h^{2}\left(\begin{array}{c}
-\frac{863}{10080} \\
-\frac{271}{10080} \\
-\frac{1}{126} \\
-\frac{39}{1120} \\
\frac{16}{315}
\end{array}\right)\left(g_{n+5}\right)
$$

using (16) to obtain the block solution as

$$
\begin{aligned}
& \left(\begin{array}{lllll}
1 & 0 & 0 & 0 & 0 \\
0 & 1 & 0 & 0 & 0 \\
0 & 0 & 1 & 0 & 0 \\
0 & 0 & 0 & 1 & 0 \\
0 & 0 & 0 & 0 & 1
\end{array}\right)\left(\begin{array}{l}
y_{n+1} \\
y_{n+2} \\
y_{n+3} \\
y_{n+4} \\
y_{n+5}
\end{array}\right) \\
& =\left(\begin{array}{l}
1 \\
1 \\
1 \\
1 \\
1
\end{array}\right)\left(y_{n}\right)+h\left(\begin{array}{ccccc}
\frac{4919}{4480} & -\frac{6347}{7520} & \frac{2563}{3360} & -\frac{307}{560} & \frac{129571}{604800} \\
\frac{3797}{2520} & -\frac{38}{945} & \frac{283}{630} & -\frac{227}{630} & \frac{5489}{37800} \\
\frac{6567}{4480} & \frac{127}{280} & \frac{1233}{1120} & -\frac{291}{560} & \frac{4393}{22400} \\
\frac{52}{35} & \frac{344}{945} & -\frac{176}{105} & \frac{2}{35} & \frac{548}{4725} \\
\frac{11875}{8064} & \frac{625}{1512} & \frac{3125}{2016} & \frac{625}{1008} & \frac{15515}{24192}
\end{array}\right)\left(\begin{array}{l}
f_{n+1} \\
f_{n+2} \\
f_{n+3} \\
f_{n+4} \\
f_{n+5}
\end{array}\right) \\
& +h\left(\begin{array}{c}
\frac{2627}{8400} \\
\frac{943}{3150} \\
\frac{849}{2800} \\
\frac{158}{525} \\
\frac{305}{1008}
\end{array}\right)\left(f_{n}\right)+h^{2}\left(\begin{array}{c}
-\frac{863}{10080} \\
-\frac{37}{630} \\
-\frac{87}{1120} \\
-\frac{16}{315} \\
\frac{275}{2016}
\end{array}\right)\left(g_{n+5}\right)
\end{aligned}
$$

Written the above explicitly gives:

$$
\begin{aligned}
y_{n+1}= & y_{n}+h\left[\frac{2627}{8400} f_{n}+\frac{4919}{4480} f_{n+1}-\frac{6347}{7560} f_{n+2}+\frac{2563}{3360} f_{n+3}\right. \\
& \left.-\frac{307}{560} f_{n+4}+\frac{129571}{604800} f_{n+5}\right]-\frac{863}{10080} h^{2} g_{n+5} \\
y_{n+2}= & y_{n}+h\left[\frac{943}{3150} f_{n}+\frac{3797}{2520} f_{n+1}-\frac{38}{945} f_{n+2}+\frac{283}{630} f_{n+3}\right. \\
& \left.-\frac{227}{630} f_{n+4}+\frac{5489}{37800} f_{n+5}\right]-\frac{37}{630} h^{2} g_{n+5}
\end{aligned}
$$




$$
\begin{aligned}
y_{n+3}= & y_{n}+h\left[\frac{849}{2800} f_{n}+\frac{6567}{4480} f_{n+1}+\frac{127}{280} f_{n+2}+\frac{1233}{1120} f_{n+3}\right. \\
& \left.-\frac{291}{560} f_{n+4}+\frac{4393}{22400} f_{n+5}\right]-\frac{87}{1120} h^{2} g_{n+5} \\
y_{n+4}= & y_{n}+h\left[\frac{158}{525} f_{n}+\frac{52}{35} f_{n+1}+\frac{344}{945} f_{n+2}+\frac{176}{105} f_{n+3}\right. \\
& \left.+\frac{2}{35} f_{n+4}+\frac{548}{4725} f_{n+5}\right]-\frac{16}{315} h^{2} g_{n+5} \\
y_{n+5}= & y_{n}+h\left[\frac{305}{1008} f_{n}+\frac{11875}{8064} f_{n+1}+\frac{625}{1512} f_{n+2}+\frac{3125}{2016} f_{n+3}\right. \\
& \left.+\frac{625}{1008} f_{n+4}+\frac{15515}{24192} f_{n+5}\right]-\frac{275}{2016} h^{2} g_{n+5}
\end{aligned}
$$

\section{Analysis and Implementation of the Method}

\section{Order, Error Constant and Consistency of the Method}

Basic properties of the main schemes and their associated block schemes, are analysed to establish their validity. These properties, namely: order, error constant, consistency and zero stability reveal the nature of convergence of the schemes. The region of absolute stability of the methods have also been obtained in this section.

\section{Order of the Scheme}

Let the linear difference operator $L$ associated with the continuous multiderivative method (2) be defined as

$$
\begin{aligned}
& L[y(x) ; h] \\
& =\sum_{j=0}^{k}\left\{\alpha_{j} y\left(x_{n}+j h\right)-h \beta_{j} y^{\prime}\left(x_{n}+j h\right)-h^{2} \beta_{j} y^{\prime \prime}\left(x_{n}+j h\right) ; j=1,2, \cdots, m\right\}
\end{aligned}
$$

where $y(x)$ is an arbitrary test function that is continuously differentiable in the interval $[a, b]$. Expanding $y\left(x_{n}+j h\right)$ and $y^{\prime \prime}\left(x_{n}+j h\right), j=0,1,2, \cdots$ in Taylor series about $x_{n}$ and collecting like terms in $h$ and $y$ gives:

$$
L[y(x) ; h]=C_{0} y(x)+C_{1} h y^{(1)}(x)+C_{2} h^{2} y^{(2)}(x)+\cdots+C_{p} h^{p} y^{(p)}(x)+\cdots
$$

Definition (4.1.1). The difference operator $L$ and the associated continuous multistep method (2) are said to be of order $p$

$$
C_{0}=C_{1}=C_{2}=C_{3}=\cdots=C_{p}=C_{p}=0, C_{p+1} \neq 0 \text { Lambert [10]. }
$$

\section{Order of the Block Scheme}

The order of the block will be defined following the method of Chollom [11].

Let the multi-derivative method be defined by:

$$
\sum_{j} \alpha_{i j}^{(\mu)}(t) y_{n+j}=h \sum_{j} \beta_{i j}^{(\mu)}(t) f_{n+j}+h^{2} \sum_{j} \gamma_{i j}^{(\mu)}(t) g_{n+j},
$$

where $\mu$ is the degree of the derivative of the continuous cofficients $\alpha_{i j}(t)$, $\gamma_{i j}$ and $\beta_{i j}(t)$. 
The linear difference operator, $\mathrm{L}$, associated with the implicit block hybrid one step method (15) is described by the formula:

$$
\begin{aligned}
& L[y(x) ; h] \\
& =\sum_{j}\left[\bar{\alpha}_{j} y\left(x_{n}+j h\right)-h \bar{\beta}_{j} y^{\prime}\left(x_{n}+j h\right)-h^{2} \gamma_{j}\left(x_{n}+j h\right)\right]
\end{aligned}
$$

where $y(x)$ is an arbitrary test function continuously differentiable on $[a, b]$. Expanding $y\left(x_{n}+j h\right)$ and $y^{\prime \prime}\left(x_{n}+j h\right)$, in Taylor series and collecting terms in (41) gives:

$$
L[y(x) ; h]=\bar{C}_{0} y(x)+\bar{C}_{1} h y^{(1)}(x)+\bar{C}_{2} h^{2} y^{(2)}(x)+\cdots+\bar{C}_{p} h^{p} y^{(p)}(x)
$$

where the $\bar{C}_{i}, i=0,1, \cdots, p$ are vectors.

\section{Consistency}

Given a continuous multi-derivative method (2), the first and second characteristic polynomials are defined as

$$
\begin{gathered}
\rho(z)=\sum_{j=0}^{k} \alpha_{j} z^{j} \\
\sigma(z)=\sum_{j=0}^{k} \beta_{j} z^{j}
\end{gathered}
$$

where $z$ is the principal root, $\alpha_{k} \neq 0$ and $\alpha_{0}^{2}+\beta_{0}^{2} \neq 0$.

Definition (4.1.2). The continuous multi-derivative method (2) is said to be consistent if it satisfies the following conditions:

1) the order $p \geq 1$;

2) $\sum_{j=0}^{k} \alpha_{j}=0$;

3) $\rho(1)=\rho^{\prime}(1)=0$;

4) $\rho^{\prime \prime}(1)=2 ! \sigma(1)$.

Definition (4.1.3). According to Gurjinder [12]. A block method is said to be consistent if it has an order of convergence, say $p \geq 1$.

Applying the definitions for order of the method on the main scheme (11) for four-step method and order of the block methods on (18)-(21) likewise order of the main scheme (27) for five-step method and order of the block methods on (32)-(36) it was derived that the main scheme (11) is of order $p=6$ with Error Constant $C_{p+1}=\frac{41}{30240}$ and the additional schemes are of uniform order $p=6$, likewise for the five-step method the main scheme (27) is of order $p=7$ with Error Constant $C_{p+1}=\frac{731}{846720}$. And the additional schemes is of uniform order $p=7$. The order of four-step and five-step block methods are in tabular form as indicated in Table 1 and Table 2.

The main schemes and additional schemes for four-step method and five-step method are consistent since they have order $p \geq 1$.

\section{Zero Stability and Convergent}

It is known from literature that the stability of linear multi-step method determine the manner in which the error is propagated as the numerical computation 
Table 1. Order and error constants for Block methods for $k=4$.

\begin{tabular}{ccc}
\hline Method & Order & Error Constant $C_{p+1}$ \\
\hline$(18)$ & 6 & $-\frac{8}{945}$ \\
$(19)$ & 6 & $-\frac{11}{1120}$ \\
$(20)$ & 6 & $-\frac{1}{126}$ \\
$(21)$ & 6 & $-\frac{3327}{30240}$ \\
\hline
\end{tabular}

Table 2. Order and error constants for Block methods for $k=5$.

\begin{tabular}{ccc}
\hline Method & Order & Error Constant $C_{p+1}$ \\
\hline$(32)$ & 7 & $\frac{1375}{169344}$ \\
$(33)$ & 7 & $\frac{16}{2205}$ \\
$(34)$ & 7 & $\frac{257}{31360}$ \\
$(35)$ & 7 & $\frac{187}{26460}$ \\
$(36)$ & 7 & $\frac{2633}{282240}$ \\
\hline
\end{tabular}

proceeds hence the investigation of the zero-stability property is necessary.

Definition (4.1.4). The continuous multi-derivative method (2) is said to be zero-stable if no root of the first characteristic polynomial $\rho(z)$ has modulus greater than one, and if every root of modulus one has multiplicity not greater than one.

Definition (4.1.5). The implicit hybrid block method (17) is said to be zero stable if the roots $Z_{s}, s=1, \cdots, n$ of the first characteristic polynomial $\bar{\rho}(z)$, defined by

$$
\bar{\rho}(z)=\operatorname{det}[z \bar{A}-\bar{E}]
$$

satisfies $\left|z_{s}\right| \leq 1$ and every root with $\left|z_{s}\right|=1$ has multiplicity not exceeding two in the limit as $h \rightarrow 0$. Lambert [10] The convergence of the continuous multi-derivative method (2) is considered in the light of the basic properties discussed earlier in conjunction with the fundamental theorem of Dahlquist (Henrici, [13]) for linear multistep methods. In what follows, we state Dahlquist's theorem without proof. The investigation carried out on the main schemes, additional schemes and block methods for four-step method and five-step 
method revealed that all the derived schemes are less than or equal to 1 hence the schemes are zero-stable. Since the consistency and zero-stability of all the schemes have been established hence, the proposed schemes are converges.

\section{Numerical Experiments}

In order to study the efficiency of the developed schemes, we present some numerical experiments with the following five examples. The Four-Step Methods (4step) and Five-Step Methods (5step) were applied to solve the following test problems:

Example 1. The linear problem by Enright in Ehigie [5],

$$
\begin{gathered}
y_{1}=-0.1 y_{1}, \quad y_{1}(0)=1 \\
y_{2}=-10 y_{2}, y_{2}(0)=1 \\
y_{3}=-100 y_{3}, y_{3}(0)=1 \\
y_{4}=-1000 y_{4}, y_{4}(0)=1
\end{gathered}
$$

is solved in the range $0 \leq x \leq 10$.

Exact solution: $y_{1}=\exp (-0.10 x), \quad y_{2}=\exp (-10 x), \quad y_{3}=\exp (-100 x)$, $y_{4}=\exp (-1000 x)$.

\section{Example 2.}

Considering a real life problem, which is an epidemical model (SIR model) that computes the theoretical numbers of people infected with a contagious illness in a closed population over time. The name of this class of models derive from the fact that they involves couples equations relating the numbers of susceptiple people $S(t)$, number of people infected $I(t)$, number of people who have recovered $R(t)$. This is a good and simple model for many infectious desease including measles, mumps and rubella Sunday [14]. The SIR model is described by the three coupled equations.

$$
\begin{gathered}
\frac{\mathrm{d} s}{\mathrm{~d} t}=\mu(I-S)-\beta I S \\
\frac{\mathrm{d} I}{\mathrm{~d} t}=-\mu I-\gamma I+\beta I S \\
\frac{\mathrm{d} R}{\mathrm{~d} t}=-\mu R+\gamma I
\end{gathered}
$$

where $\mu, \gamma$ are positive parameters. Defined y to be

$$
y=S+I+R
$$

Adding Equations (45) to (47) to obtain the following evolution equation for $y$

$$
y^{\prime}=\mu(1-y)
$$

taking $\mu=0.5$ and attaching initial condition $y(0)=0.5$ (for a particular closed population) to obtain, 


$$
y^{\prime}(t)=0.5(1-y), y(0)=0.5
$$

whose exact solution is $y(t)=1-0.5 \mathrm{e}^{-0.5 t}$.

\section{Example 3.}

The non-linear system solved by $\mathrm{Wu}$ and Xia cited in Ehigie [5], is considered.

$$
\begin{gathered}
y^{\prime}=-1002 y_{1}+1000 y_{2}^{2}, \quad y_{1}(0)=1, \\
y_{2}^{\prime}=y_{1}-y_{2}\left(1+y_{2}\right), y_{2}(0)=1
\end{gathered}
$$

Exact solution $y_{1}(x)=\mathrm{e}^{-2 x}, \quad y_{2}(x)=\mathrm{e}^{-x}$

\section{Example 4.}

Considering the moderately stiff problem solve by Jia-Xiang and Jiao-Xun cited in Ehigie [5],

$$
\begin{aligned}
& y^{\prime}=-y-10 z, \quad y(0)=1, \\
& z^{\prime}=-10 y-z, \quad z(0)=0,
\end{aligned}
$$

Exact solution $y(x)=\mathrm{e}^{-x} \cos 10 x, \quad z(x)=\mathrm{e}^{-x} \sin 10 x$

\section{Discussion of Results}

In this work, new multi-derivative multistep methods of order $(6,7)$ are proposed for the solution of first order Initial Value Problems. The main method and additional methods for cases $(k=4,5)$ are obtained from the same continuous scheme derived via interpolation and collocation procedures. The stability properties and region of the method were also discussed. The methods were then applied in block form as simultaneous numerical integrators over non-overlapping interval. Numerical results obtained using the proposed block form show that it is attractive for the solution of linear and non-linear problems.

In Tables 3-7, numerical examples were used to implement the new derived methods, comparing the accuracy of method with those of Ehigie, Okunuga Jator and Sofoluwe [5], Wu and Xia, Jia-Xiang and Jiao-Xun, Enright [3] cited in ehigie [5] and a real life problem in sunday [14]. It can be observed that the new studies display better accuracy than most of the existing methods considered.

Table 3. Comparison of errors of the new method with exixting one for Example 1 when $h=0.01$.

\begin{tabular}{cccccc}
\hline \multicolumn{1}{c}{ Step } & $y_{1}$ & $y_{2}$ & $y_{3}$ & $y_{4}$ \\
\hline Enright [3] & 1000 & $4.1 \times 10^{-7}$ & - & - & - \\
Ehigie [5] & 1000 & $4.2 \times 10^{-16}$ & $2.1 \times 10^{-10}$ & 0 & 0 \\
Four-Step & 1000 & 0 & 0 & 0 & 0 \\
Five-Step & 1000 & 0 & 0 & 0 & 0 \\
\hline
\end{tabular}


Table 4. Comparison of error of the new methods with existing ones for Example 2 when $h=0.1$.

\begin{tabular}{cccc}
\hline$x$ & Four-Step & Five-Step & Sunday [14] \\
\hline 0.1 & $3.766 \times 10^{-12}$ & $1.54 \times 10^{-13}$ & $2.0 \times 10^{-11}$ \\
0.2 & $2.498 \times 10^{-12}$ & $1.08 \times 10^{-13}$ & $3.0 \times 10^{-11}$ \\
0.3 & $3.013 \times 10^{-12}$ & $1.23 \times 10^{-13}$ & $1.0 \times 10^{-10}$ \\
0.4 & $2.408 \times 10^{-12}$ & $1.00 \times 10^{-13}$ & $2.0 \times 10^{-10}$ \\
0.5 & $5.374 \times 10^{-12}$ & $1.09 \times 10^{-13}$ & $1.0 \times 10^{-10}$ \\
0.6 & $4.225 \times 10^{-12}$ & $2.24 \times 10^{-13}$ & $2.0 \times 10^{-10}$ \\
0.7 & $4.538 \times 10^{-12}$ & $1.82 \times 10^{-13}$ & $1.0 \times 10^{-10}$ \\
0.8 & $3.943 \times 10^{-12}$ & $1.90 \times 10^{-13}$ & $2.0 \times 10^{-10}$ \\
0.9 & $6.274 \times 10^{-12}$ & $1.67 \times 10^{-13}$ & $3.0 \times 10^{-10}$ \\
1.0 & $5.242 \times 10^{-12}$ & $1.70 \times 10^{-13}$ & $3.0 \times 10^{-10}$ \\
\hline
\end{tabular}

Table 5. Comparison of error of the new methods with existing one for Example 3.

\begin{tabular}{ccccc}
\hline $\begin{array}{c}\text { No. of } \\
\text { Steps }\end{array}$ & $\begin{array}{c}\text { Four-Step } \\
\max \left\|y_{i}-y\left(x_{i}\right)\right\|\end{array}$ & $\begin{array}{c}\text { Four-Step } \\
\max \left\|z_{i}-z\left(x_{i}\right)\right\|\end{array}$ & $\begin{array}{c}\text { Ehigie [5] } \\
\max \left\|y_{i}-y\left(x_{i}\right)\right\|\end{array}$ & $\begin{array}{c}\text { Ehigie [5] } \\
\max \left\|z_{i}-z\left(x_{i}\right)\right\|\end{array}$ \\
\hline 125 & $1.121 \times 10^{-11}$ & $7.224 \times 10^{-11}$ & $8.33 \times 10^{-06}$ & $1.32 \times 10^{-6}$ \\
250 & $1.225 \times 10^{-13}$ & $3.551 \times 10^{-13}$ & $1.13 \times 10^{-07}$ & $1.36 \times 10^{-08}$ \\
500 & $1.00 \times 10^{-15}$ & $6.780 \times 10^{-15}$ & $8.19 \times 10^{-12}$ & $6.30 \times 10^{-12}$ \\
\hline
\end{tabular}

Table 6. Comparison of error of the new methods with existing one for Example 3.

\begin{tabular}{|c|c|c|c|c|}
\hline $\begin{array}{l}\text { No. of } \\
\text { Steps }\end{array}$ & $\begin{array}{c}\text { Five-Step } \\
\max \left\|y_{i}-y\left(x_{i}\right)\right\|\end{array}$ & $\begin{array}{c}\text { Five-Step } \\
\max \left\|z_{i}-z\left(x_{i}\right)\right\| \\
\left\|z_{i}-z\left(x_{i}\right)\right\|\end{array}$ & $\begin{array}{c}\text { Ehigie [5] } \\
\max \left\|y_{i}-y\left(x_{i}\right)\right\|\end{array}$ & $\begin{array}{c}\text { Ehigie [5] } \\
\max \left\|z_{i}-z\left(x_{i}\right)\right\|\end{array}$ \\
\hline 125 & $1.635 \times 10^{-12}$ & $4.795 \times 10^{-12}$ & $8.33 \times 10^{-06}$ & $1.32 \times 10^{-6}$ \\
\hline 250 & $2.0 \times 10^{-15}$ & $1.6 \times 10^{-14}$ & $1.13 \times 10^{-07}$ & $1.36 \times 10^{-08}$ \\
\hline 500 & 0 & 0 & $8.19 \times 10^{-12}$ & $6.30 \times 10^{-12}$ \\
\hline
\end{tabular}

Table 7. Comparison of error of the new methods with existing one for Example 4.

\begin{tabular}{cccc}
\hline Method & Number of Steps & $\max \left\|y_{i}-y\left(x_{i}\right)\right\|$ & $\max \left\|z_{i}-z\left(x_{i}\right)\right\|$ \\
\hline Wu-Xia [5] & 500 & $2.56 \times 10^{-07}$ & $8.02 \times 10^{-08}$ \\
Four-Step & 500 & $5.68 \times 10^{-09}$ & $5.38 \times 10^{-09}$ \\
Five-Step & 500 & $4.22 \times 10^{-13}$ & $9.89 \times 10^{-13}$ \\
\hline
\end{tabular}




\section{Funding}

The study was not funded by any grant, it was funded by the authors.

\section{Conflicts of Interest}

The authors declare that there is no conflict of interest.

\section{References}

[1] Onumanyi, P. Awoyemi, D.O., Jator, S.N. and Sirisena, U.W. (1994) New Linear Multistep Methods with Continuous Coefficients for First Order Initial Value Problems. Journal of the Nigerian Mathematical Society, 13, 37-51

[2] Fox, L. (1954) A Note on the Numerical Integration of First-Order Differential Equations. Quarterly Journal of Mechanics and Applied Mathematics, 7, 367-378. https://doi.org/10.1093/qjmam/7.3.367

[3] Enright, W.H. (1974) Second Derivative Multistep Methods for Stiff Ordinary Differential Equations. SIAM Journal on Numerical Analysis, 11, 321-331.

https://doi.org/10.1137/0711029

[4] Cash, J.R. (1981) On the Exponential Fitting of Composite Multiderivative Linear Multistep Methods. SIAM Journal on Numerical Analysis, 18, 808-821. https://doi.org/10.1137/0718055

[5] Ehigie J.O., Jator S.N., Sofoluwe, A.B. and Okunuga, S.A. (2014) Boundary Value Technique for Initial Problems with Continuous Second Derivative Multistep Method of Enright. SIAM Journal on Numerical Analysis, 33, 81-93. https://doi.org/10.1007/s40314-013-0044-4

[6] Axelsson, A.O.H. and Verwer, J.G. (1985) Boundary Value Techniques for Initial Value Problems in Ordinary Differential Equation. Mathematics of Computation, 45, 151-171. https://doi.org/10.1090/S0025-5718-1985-0790649-9

[7] Amodio, P. and Mazzia, F. (1995) Variable Step Boundary Value Methods Based on Reverse Adams Schemes and Grid Distribution. Applied Numerical Mathematics, 18, 5-21. https://doi.org/10.1016/0168-9274(95)00044-U

[8] Brugano, L. and Trigiante, D. (1998) Solving Differential Problems by Multistep Initial and Boundary Value Methods. Gordon and Breach Science Publisher, Amsterdam.

[9] Jator, S.N. and Sahi, R.K. (2010) Boundary Value Techniques for Initial Value Problems Based on Adams-Type Second Derivative Methods. International Journal of Mathematical Education in Science and Technology, 41, 819-826. https://doi.org/10.1080/00207391003675141

[10] Lambert, J.D. (1973) Computational Methods in Ordinary Differential Equations. John Willey \& Sons, New York.

[11] Chollom, J.P., Ndam, J.N. and Kumleng, G.M. (2007) On Some Properties of the Block Linear Multistep Methods. Science World Journal, 2, 11-17. https://doi.org/10.4314/swj.v2i3.51747

[12] Gurjinder, S., Kanwar, V. and Saurabh, B. (2013) Exponentially Fitted Variants of the Two-Step Adams-Bashforth Method for the Numerical Integration of Initial Value Problem. Journal of Application and Applied Mathematics, 8, 741-755.

[13] Henrici, P. (1962) Discrete Variable Method for Ordinary Differential Equations. John Wiley \& Sons, UK. 
[14] Sunday, J., Odekunle, M.R. and Adesanya, A.O. (2013) Order Six Block Integrator for the Solution of First-Order Ordinary Differential Equations. International Journal of Mathematics and Soft Computing, 3, 87-96.

https://doi.org/10.26708/IJMSC.2013.1.3.10 